\title{
Development of an Intuitive Interface for PC Mouse Operation Based on Both Arms Gesture
}

\author{
Nobuaki Nakazawa ${ }^{1 *}$, Toshikazu Matsui ${ }^{1}$, Yusaku Fujii ${ }^{2}$ \\ ${ }^{1}$ Faculty of Science and Technology, Gunma University, 29-1 Ota, Gunma 373-0057, Japan. \\ 2 Faculty of Science and Technology, Gunma University, 1-5-1 Kiryu, Gunma 376-8515, Japan. \\ * Corresponding author. Tel.: +81-276-50-2244; email: n.nakazawa@gunma-u.ac.jp \\ Manuscript submitted June 15, 2017; accepted July 10, 2017. \\ doi: $10.17706 /$ jsw.13.8.453-459
}

\begin{abstract}
This paper describes an intuitive interface to operate the mouse based on both arm gestures. The Kinect sensor provided the skeleton information of the user's posture, which was used for detection of joint positions of upper half of body. From the obtained joint positions, the motion of the operator was recognized to reflect a mouse operation. Here, we constructed the system which could choose two displays, scroll and move the position of the mouse cursor by changing input modes according to the length of the extended right hand. The user could decide the position of the cursor with the right hand, and click with the left hand. In addition, we made the wearable device on the wrist for sensory feedback to user. In the test operation of the developed system, the user could successfully operate the mouse cursor by using both arms gesture, as same as the general mouse and the touch pad device.
\end{abstract}

Key words: Gesture, human interface, kinect sensor, arm, operation.

\section{Introduction}

When we communicate with someone, the interaction with body and hand gestures are sometimes used in addition to words. People who saw it can easily recognize and understand the opponents' will by movement. Such gestures have the potential as an interface that connects humans and machines, and so far, sign language [1], [2], finger letters [3], handed gestures [4]-[7], and head recognition of department gestures [8], have been studied from various view point. Also, the system development such as operation of home appliances using gestures [9], [10] and operation of wheelchairs [11]-[13] have been carried out. Operation of equipment based on gesture does not require touching the device, it can operate intuitively. If gesture is applied to PC operation, body posture is not restrained such as touching keyboard and mouse, so it seems to be effective for the person who cannot use the hand to operate a personal computer. This paper proposed an intuitive interface to operate the mouse cursor of a personal computer based on both arm gestures. The Kinect sensor provided the skeleton information of the user's posture, which was used for detection of joint positions of upper half of body. From the obtained joint positions, the motion of the operator was recognized to reflect a mouse operation. Here, we constructed the system, which could choose two displays, scroll and move the position of the mouse cursor by changing the functions according to the length of the extended right hand.

\section{Apparatus}

In the medical field such as the surgery by a doctor and the dental treatment by a dentist, the medical thin 
gloves that is worn in the hand must be clean hygienically because the medical thin glove is directory contacted with the affected part of a patient. However, it is often necessary to use a personal computer even during treatment tasks. In the X-ray room, the doctor sometimes treats multiple displays not only to see the X-ray scan image but also to input the personal computer. Here, we consider the situation that the users operate multi personal computers at the same time, without handling the keyboard and the mouse devises. As shown in Fig.1, we prepared two computer that were operated by one user. In order to avoid the hand touch with the device, we focused on the dynamic gesture. The Kinect sensor (Microsoft L6M-00020) was used for the measurement of the user's arm position in three dimensions to recognize the user's gesture intention. The obtained intention was reflected on the personal computer operations. The LED tape was put on each display to indicate the selected computer.

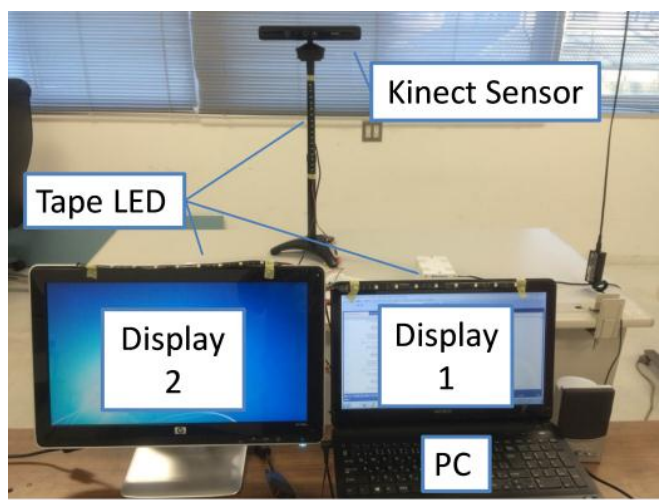

Fig. 1. Control system with two displays and the Kinect sensor to recognize the user's gesture.

\section{Gesture Assignment}

\subsection{Operation Layers}

In this interface, in order to realize a plurality of operations, the right arm was captured in three dimensions. The captured hand range was divided into three by the distance from the shoulder to the tip of the hand, three layers were made, and the input mode was changed in each area. Fig.2 shows a schematic diagram of the input mode set for each of the three layers. Also, in order to confirm the position of the right

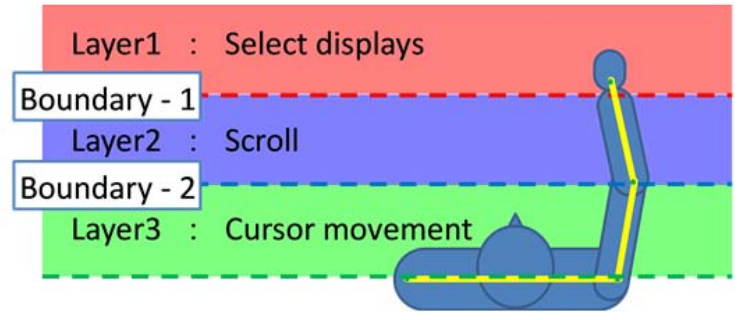

Fig. 2. Three layers for operation.

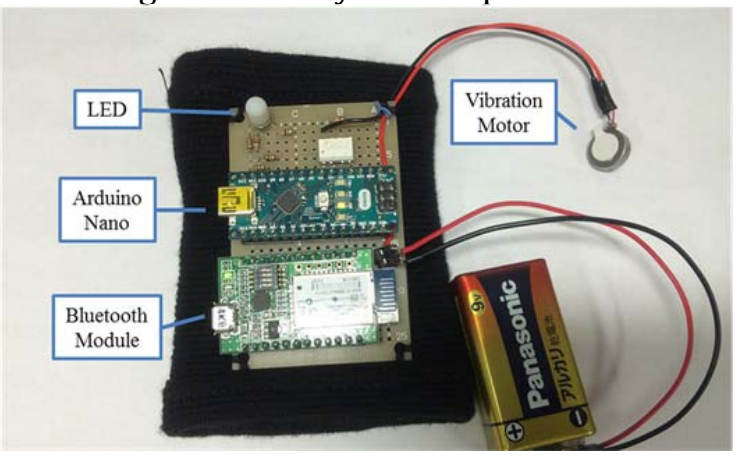

Fig. 3. The feedback device.
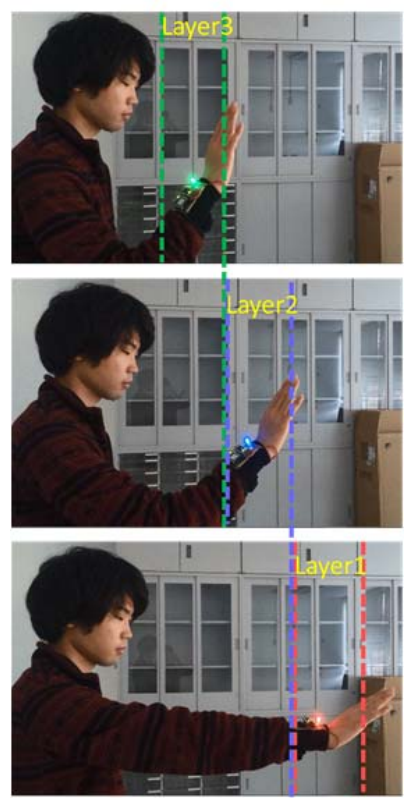

Fig. 4. Scene of LED illumination. 
hand in the layer divided into three by the operator, the full color LED of the feedback device shown in Fig. 3 was lit in a color corresponding to each layer. At the same time, the vibration motor vibrated, thereby notifying the user that the layer had been changed. The control signal was transfer from the personal computer connecting with the Kinect sensor through the microcomputer (Arduino Nano). Fig.4 shows the scene of the LED illumination when the user extended the upper limb to select each layers. The selection of the screen was done by pointing at the display of the person who wishes to select on the most back layer 1 (Layer 1) where the arm is extended far. The scrolling operation was performed by a gesture that rotates the hand by the right hand within the range of the middle layer 2 (Layer 2). Further, the operation of moving the mouse cursor was reflected by the coordinates of the right hand in the range of the layer 3(Layer 3) in front. As for the click operation, it was possible to do with the left hand in all layers.

\subsection{Display Selection}

To select a screen, the user instructed the display with the finger, as shown in Fig.5. If the hand was over the Boundary-1, the display selection mode started. In this state, the user could operate the personal computers. When the right hand was on the right side with respect to the right shoulder, the right screen was selected. Likewise, when the right hand was on the left side with respect to the right shoulder, the left screen was selected. After that, the cursor position moved to the center of the selected screen, and the tape LED attached to the top of the screen as shown in Fig.1 illuminated to indicate the selected active display.

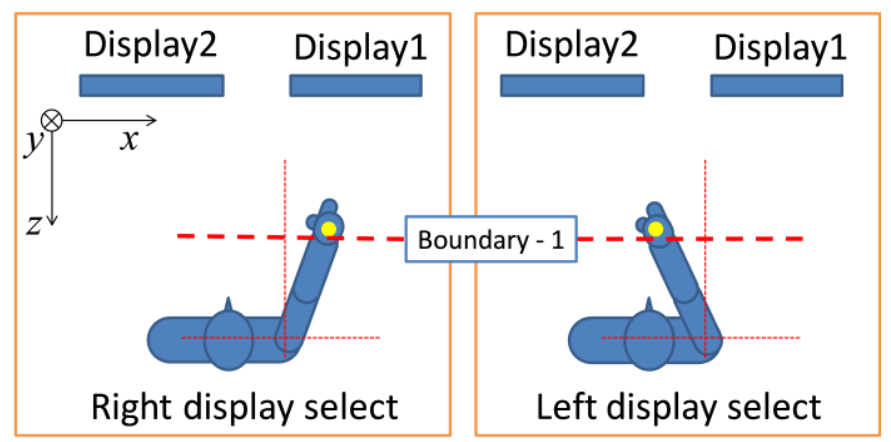

Fig. 5. Display selection.

\subsection{Cursor Position}

The position of the user's right hand was obtained from the Kinect sensor and it was used for the cursor position. Fig. 6 shows the correspondence between the human hand position and the cursor coordinates displayed on the screen. The figure is mirrored, and the left and right hands are reversed. Fig.6 (a) shows the cases where the hand was located within the virtual control circle displayed by the dotted circle in this figure. In this case, the hand position was directly converted to the mouse cursor position. The relational expression between the hand position $\left(u_{(t)}, v_{(t)}\right)$ at time $t$ detected by the Kinect sensor and the

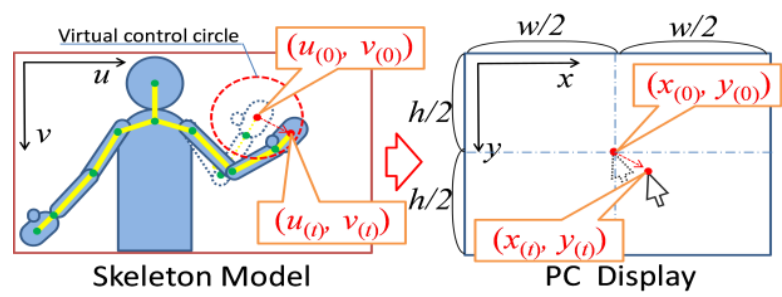

(a) Within control circle

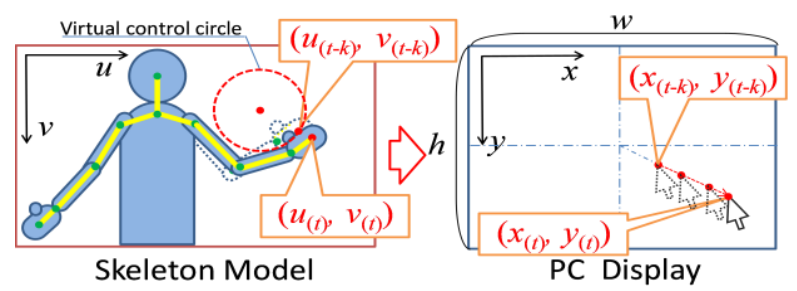

(b) Outside control circle

Fig. 6. Mouse cursor operation and virtual control circle. 
coordinates $\left(x_{(t)}, y_{(t)}\right)$ of the mouse cursor was as follows.

$$
\left\{\begin{array}{l}
x_{(t)}=w / 2+\xi\left(u_{(t)}-u_{(0)}\right) \\
y_{(t)}=h / 2+\xi\left(v_{(t)}-v_{(0)}\right)
\end{array}\right.
$$

where, parameter $\xi$ is an arbitrary magnification. With the center $(w / 2, h / 2)$ of the screen as a reference, the displacement from the coordinates $\left(u_{(0)}, v_{(0)}\right)$ of the hand position at the start of the synchronization of the movement of the hand and the cursor is multiplied by the magnification $\xi$ to calculate the mouse cursor position. Fig.6 (b) shows the cases where the hand was over the virtual control circle. At this moment, the vibration motor on the feedback device shown in Fig.2 vibrated to notify the user. In this case, the mouse cursor acted just like a joystick. The mouse cursor was as follows.

$$
\left\{\begin{array}{l}
x_{(t)}=x_{(t-k)}+\xi^{\prime}\left(u_{(t)}-u_{(t-k)}\right) k \\
y_{(t)}=y_{(t-k)}+\xi^{\prime}\left(v_{(t)}-v_{(t-k)}\right) k
\end{array}\right.
$$

Where, the parameter $\xi^{\prime}$ is an arbitrary magnification. At time $t-k$, the hand crossed the virtual circle. The farther the coordinates of the right hand moved away from the circle, the faster the mouse cursor moved forward to the direction with the angle $\phi$ shown by

$$
\phi=\tan ^{-1}\left\{v_{(t)}-v_{(t-k)} / u_{(t)}-u_{(t-k)}\right\} .
$$

\subsection{Click Actions}

The position of the user's left hand was assigned to the click actions. Fig.7 shows the assignment of the left hand motion to the click actions. As in Fig.6, this figure is also mirrored. Left and right clicking could be done by raising the left hand at the left and right side with respect to the left elbow, respectively. Also, left double-click worked by raising the left hand above the left shoulder. Drag operation could be performed by operating the cursor with the right hand, while the left hand was raising above the left elbow.
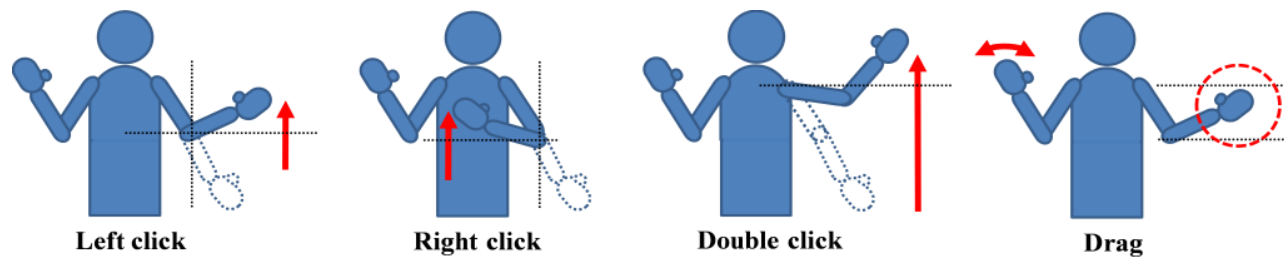

Fig. 7. Mouse cursor position derived from the right hand position of the user.

\section{Experiment}

In order to evaluate the developed gesture system, we carried out the experiment by comparing with the general mouse device and touch pad device. Fig.8 shows the experimental situation. The participated subject sat on the chair and operated the personal computer with the general mouse and the touch pad devices. The developed system was controlled by gesture with standing posture. Fig. 9 shows the target on the display. Five rectangle targets were prepared for positioning and moving the mouse cursor. The number shown in this figure was the order of movement. Twelve subjects participated in this experiment. Firstly, we evaluated the operability of the display selection. Fig.10 shows the time duration when all subjects switched the display nine times. In the case of using the touch pad, it tended to take a long time. The time duration by using the developed system was almost same as the time duration by using the general mouse. As for subject C, D and E, input by the developed system could select the display faster than other two devices. 
Next, we evaluated the mouse cursor trajectories on the display. Fig.11 shows typical results of three devices. With reference to the case of the general mouse and the touch pad device, the human subject tended to move the cursor almost linearly with minimal path from previous target point to next target one. The mouse cursor movement by the developed system was also done with minimal path, and the trajectory was not disturbed but smooth.

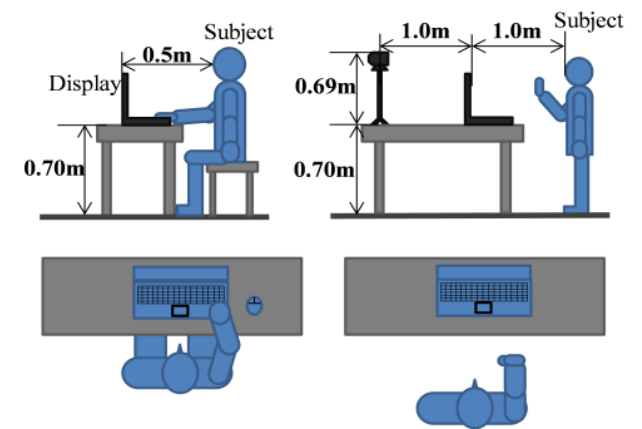

(a) Mouse, Touch Pad (b) Developed system

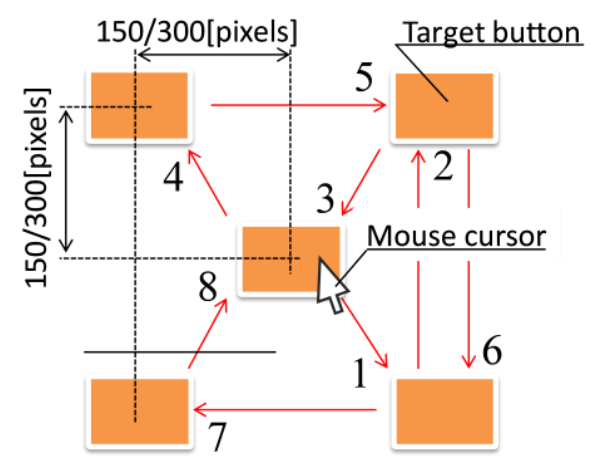

Fig. 9. Target positions on the display.

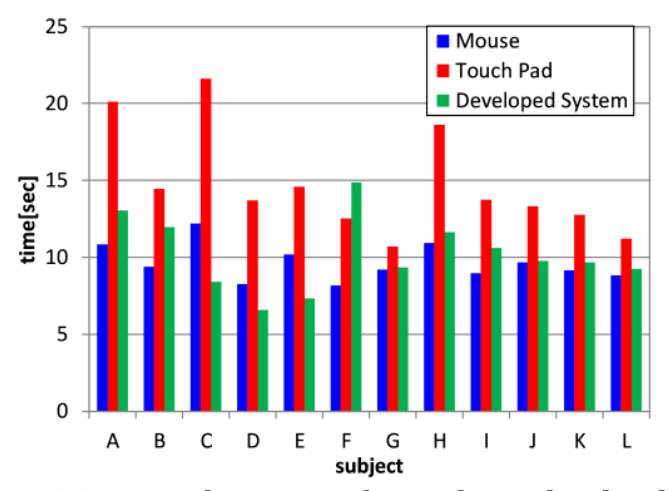

Fig. 10. Time duration of switching the display.

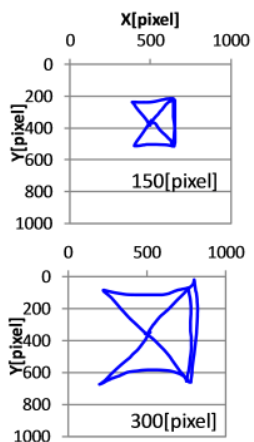

(a) Mouse

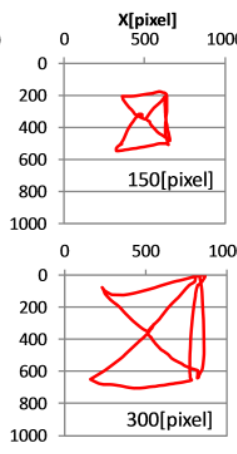

(b) Touch Pad

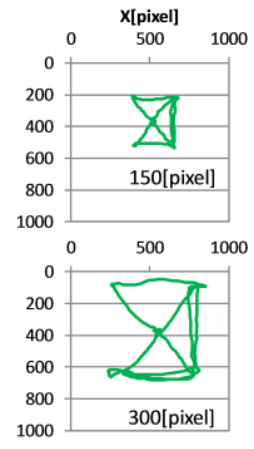

(c) Developed system

Fig. 11. Trajectories of mouse cursor position.

\section{Conclusion}

We proposed an intuitive interface to operate the PC mouse based on both arm gestures. Here, we constructed the system which could choose two displays, scroll and move the position of the mouse cursor by changing input modes according to the depth position of the right hand. The user could decide the position of the mouse cursor with the right hand, and click with the left hand. In addition, we made the wearable device on the wrist for sensory feedback to user. In the test trial of the developed system, the user could successfully operate the mouse cursor by using both arms gesture, as same as the general mouse and the touch pad device. Since this system does not touch the device with hands, it is effective for work to keep hands clean.

\section{References}

[1] Sakaguchi, T., el al. (1997). Gesture recognition using gyroscopes and accelerometers. Transactions of the Society of Instrument and Control Engineers, 33(12), 1171-1177.

[2] Fujimoto, H., et al. (2000). Trace recognition with a feature plane in the learning system of sign language. Transactions of the Japan Society of Mechanical Engineers Series C, 66(650), 3359-3365.

[3] Watanabe, K., et al. (1997), Manual alphabet recognition by using colored gloves. The transactions of 
the Institute of Electronics, Information and Communication Engineers J80-D-2(10), 2713-2722.

[4] Nishimura, T., et al.(1998), Adaptation to gesture performers by an on-line teaching system for spotting recognition of gestures from a time-varying image. The transactions of the Institute of Electronics, Information and Communication Engineers J81-D-2, 8, 1822-1830.

[5] Kirishima, T., et al. (2001), Real time gesture recognition by selective control of visual interest points. The transactions of the Institute of Electronics, Information and Communication Engineers. D-II J84-D-II, 2398-2407.

[6] Badgujar, S. D., et al. (2014), Hand gesture recognition, International Journal of Scientific and Research Publications, 4(2), 1-5.

[7] Ge, S. S., et al. (2008), Hand gesture recognition and tracking based on distributed locally linear embedding. Image and Vision Computing, 26, 1607-1620.

[8] Wu, H., et al. (1996). Head gesture recognition from time - Varying color images. Journal of Information Processing Society of Japan, 37(6), 1234-1242.

[9] Tsukada, K., et al. (2002), Ubi-Finger: Gesture input device for mobile use. Journal of information processing society of Japan, 43(12), 3675-3684.

[10] Fukumoto, M., et al. (1999), UbiButton: A bracelet style fulltime wearable commander. IPSJ Journal, 40(2), 389-398.

[11] Murashima, M., et al. (2000), Understanding and learning of gestures through Human — Machine interaction.

[12] Chitte P. P., et al. (2016), A hand gesture based wheelchair for physically handicapped person with emergency alert system, International Research Journal of Engineering and Technology, 3(4), 277-281.

[13] Landge, S., et al. (2017), Accelerometer based gesture controlled wheelchair. International Journal of Computer Applications, 161(10), 9-12.

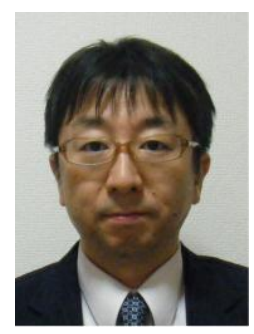

Nobuaki Nakazawa was born in 1969, Japan. He received the B.S. degree from Toyama University in 1993, and M.S and Dr. Eng. degrees from Tohoku University, Japan in 1995 and 1998, respectively. From 1998 to 1999, he was a research fellow of Japan Society for the Promotion Science (JSPS) at Tohoku University. From 1999 to 2006, he was a research associate of Graduate School of Engineering, Gunma University, Japan. Since 2007, he has been an associate professor of the Department of Production Science and Technology, Gunma University, Japan. His research interests include ergonomics, human interface, and welfare support-equipment.

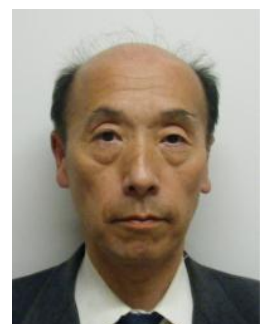

Toshikazu Matsui was born in 1954, Japan. He received the B.S. and M.S degrees from Waseda University, Japan in 1977 and 1979, respectively, and the Dr. Eng. degree from Waseda University, Japan in 1997. From 1980 to 1994, he was a Research Engineer of Toshiba Corporation. From 1994 to 1996, he was an assistant research Engineer of Advanced Telecommunications Research Institute International (ATR). From 1996 to 1998, he was a research engineer of Toshiba Corporation. From 1998 to 2006, he has been an associate professor of Graduate School of Engineering, Gunma University, Japan. Since 2007, he has been an associate professor of the Department of Production Science and Technology, Gunma University, Japan. 


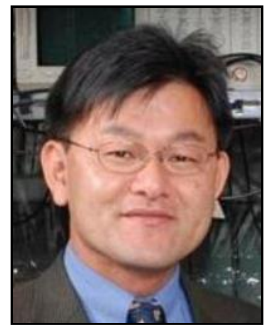

Yusaku Fujii was born in Tokyo, Japan, in 1965. He received the B.E., M.E. and Ph.D degrees from Toyko University, Tokyo, Japan, in 1989, 1991 and 2001, respectively. In 1991, he joined the Kawasaki Steel Corp. In 1995, he moved to the National Research Laboratory of Metrology (NRLM), Tsukuba, Japan, where he had studied for the replacement of the kilogram using the Superconducting Magnetic Levitation. In 2002, he moved to Gunma University, Kiryu, Japan, where has invented and studied for the Levitation Mass Method (LMM) as a precision force measurement method. He has also invented and studied for the e-JIKEI Network as a security camera system with privacy protection (www.e-jikei.org). 